\title{
Editorial: Next Generation Optoelectronics With Emerging Hybrid Materials
}

\author{
Rosanna Mastria ${ }^{1}$, Vincenzo Pecunia ${ }^{2}$ and Aurora Rizzo ${ }^{3 *}$ \\ ${ }^{1}$ Centre for Graphene Science, College of Engineering, Mathematics and Physical Sciences, University of Exeter, Exeter, \\ United Kingdom, ${ }^{2}$ School of Sustainable Energy Engineering, Simon Fraser University, Surrey, BC, Canada, ${ }^{3} \mathrm{CNR}$ \\ NANOTEC-Istituto di Nanotecnologia, Lecce, Italy
}

Keywords: hybrid materials, optoelectronic devices, colloidal nanocrystals, metal halide perovskite, graphene electrode

Editorial on the Research Topic

Editorial: Next Generation Optoelectronics With Emerging Hybrid Materials

Over the last couple of decades, the development of innovative hybrid materials, spanning from colloidal nanocrystals to hybrid halide perovskites, has offered novel and unpredictable functionalities. This fact has stimulated the development of optoelectronic devices with the potential of overcoming technological and cost constraints of current inorganic counterparts.

In this research topic, we have collected a selection of both original research and review articles covering different hybrid material synthesis and device application aspects. These articles also demonstrate the increasing interest in the development of emerging innovative hybrid materials for next-generation optoelectronic devices.

Thanks to their outstanding photophysical prerogatives, metal halide perovskites are among the

OPEN ACCESS

Edited and reviewed by: Antonio Abate, Helmholtz Association of German Research Centers (HZ), Germany

*Correspondence: Aurora Rizzo aurora.rizzo@nanotec.cnr.it

Specialty section: This article was submitted to Optoelectronics, a section of the journal Frontiers in Electronics

Received: 16 December 2021 Accepted: 07 January 2022 Published: 07 February 2022

Citation:

Mastria R, Pecunia $V$ and Rizzo A (2022) Editorial: Next Generation Optoelectronics With Emerging Hybrid Materials.

Front. Electron. 3:837260. doi: $10.3389 /$ felec.2022.837260 most appealing materials for innovative optoelectronic device applications. Nonetheless, the development of lead-free perovskites is a highly desirable prospect in view of a truly industrial deployment. In this frame, Veronese et al. report the development of a series of lead-free perovskite colloidal nanocrystals compositions $\mathrm{Cs}_{2} \mathrm{SnX}_{6}(\mathrm{X}=\mathrm{Br}, \mathrm{I})$ with different shapes. Tin-halide perovskitebased nanocrystals were selected as a valid alternative to lead. Since Sn (II) tends to easily oxidize into Sn (IV), tin-based perovskites suffer in general from a severe chemical instability that deteriorates their photophysical and optoelectronic properties. To overcome the oxidation issue of tin, the authors propose a modified hot-injection procedure in which Sn (II) can be replaced with Sn (IV). Varying the metal oxidation number results in the formation of a new crystal structure: the so-called vacancy-ordered double perovskite. They screen the effect of various surface ligands, finding that long-chain oleic acid ligands induce the formation of $3 \mathrm{D}$ colloidal nanocrystals, whereas shorter chain amines favour the growth of 2D nanoplatelets. A complete picture of the correlation between crystalline structure and optical properties of the as developed lead-free nanocrystal with the ligand molecule length and the nanocrystal shape is offered.

Another burgeoning family of materials that have captured considerable interest for optoelectronic applications involves quasi-zero-dimensional halide perovskite derivatives. Given the diversity of their embodiments, properties, and applications, the review article by Trifiletti et al. on the topic constitutes a particularly valuable contribution as it provides a comprehensive, crosssectional picture of the status of this area. A great deal of different materials is discussed, including lead-based compounds as well as lead-free ones-e.g., based on tin, bismuth, and antimony - while highlighting the salient similarities and differences between hybrid and fully inorganic embodiments. A particular merit of this review lies in the discussion of the materials and their properties through a uniquely comprehensive treatment of the corresponding processing methods, including the manifold 
strategies for bulk crystal growth, pelletization, and thin-film deposition. This approach allows the authors to reveal the synthesis-property relationships across the different types of quasi-zero-dimensional halide perovskite derivatives. The ensuing discussion of the device applications of this family of materials is equally valuable due to its comprehensiveness: in addition to photovoltaics, the article also covers light-emitting devices, photodetectors, lasers, radiation detectors, memristors, and thermoelectric energy harvesters. This cross-sectional treatment of the material, synthetic, and device properties of quasi-zero-dimensional halide perovskite derivatives provides a fresh and stimulating look at this family of materials, pointing out promising synthetic strategies and device applications that could realize their full optoelectronic potential.

Concerning application in the actual optoelectronic devices, a step towards the development of scalable deposition processes for perovskite is proposed by Smecca et al. A two-step Low-Vacuum Proximity-Space- Effusion (LV-PSE) technique is developed to reach high thermal energy and partial pressure of the sublimated species in the proximity of the target substrate. The crystallization of phase-pure $\mathrm{MAPbI}_{3}$ materials occurs via a two-step process: the sublimation of a compact $\mathrm{PbI}_{2}$ layer, and, upon the subsequent evaporation of methylammonium iodide, the conversion of $\mathrm{PbI}_{2}$ into perovskite occurring through an adsorption-incorporation-migration mechanism. The use of a low-vacuum chamber, at $\sim 4 \times 10^{-2} \mathrm{mbar}$, in perspective allows making the technique affordable and simpler if compared to conventional vacuum deposition methods. The as developed technique indeed was demonstrated to reduce the processing time, while preserving the perovskite material quality, corroborating the possible implementation of LV-PSE for industrial application. Proof-of-concept solar cell devices were eventually reported in the standard n-i-p geometry.

In addition to metal halide perovskites, other hybrid materials are emerging as promising options for optoelectronics as reported by Walsh et al. In this paper, the authors successfully used $\mathrm{FeCl}_{3}$ intercalated few-layers graphene as electrode in organic photovoltaics, tackling the need for alternative solutions to replace the expensive and brittle indium tin oxide (ITO) electrode. Unlike ITO, graphene exhibits extraordinary mechanical flexibility, transparency, and it is also compatible with low-cost and large-area fabrication, which are highly coveted properties for materials to be used as electrodes. Moreover, if compared to pristine few-layers graphene, $\mathrm{FeCl}_{3}$ intercalated graphene electrodes exhibit appealing properties such as p-type behaviour, two order of magnitude reduced sheets resistance and higher work function with better energy level alignment with the widely used hole transport layer PEDOT: PSS. All these properties make $\mathrm{FeCl}_{3}$ intercalated few-layers graphene a valid alternative to ITO as evinced also from the comparable power conversion efficiency of the devices prepared by using the two different electrodes. Furthermore, $\mathrm{FeCl}_{3}$ intercalated graphene electrodes provide additional advantages if compared to ITO. Being more resilient than ITO, devices with $\mathrm{FeCl}_{3}$ intercalated graphene exhibit a degradation rate that is five times lower than devices with ITO electrodes which is well known to be sensitive to the acidity of PEDOT:PSS. These results demonstrate that not only $\mathrm{FeCl}_{3}$ few-layers graphene is a valid substitute for ITO but thanks to its resilience enables more stable and durable organic photovoltaic devices highlighting the great opportunities that can be disclosed by using hybrid materials in optoelectronics.

\section{AUTHOR CONTRIBUTIONS}

All authors have contributed to assemble a list of potential authors and have outreached to them by personal invitations.

Conflict of Interest: The authors declare that the research was conducted in the absence of any commercial or financial relationships that could be construed as a potential conflict of interest.

Publisher's Note: All claims expressed in this article are solely those of the authors and do not necessarily represent those of their affiliated organizations, or those of the publisher, the editors and the reviewers. Any product that may be evaluated in this article, or claim that may be made by its manufacturer, is not guaranteed or endorsed by the publisher.

Copyright (c) 2022 Mastria, Pecunia and Rizzo. This is an open-access article distributed under the terms of the Creative Commons Attribution License (CC $B Y)$. The use, distribution or reproduction in other forums is permitted, provided the original author(s) and the copyright owner(s) are credited and that the original publication in this journal is cited, in accordance with accepted academic practice. No use, distribution or reproduction is permitted which does not comply with these terms. 